\title{
PREFACE TO THE 2013 EDITION
}

I was introduced to thinking about bad statistics when I read Darrell Huff's How to Lie with Statistics as a first-year college student. By the time I checked out the library's copy, that book was more than ten years old, and the volume showed a lot of wear. Huff's examples no longer seemed current, and I particularly remember that the graphs he reproduced from newspaper articles struck me as somehow old-fashioned. Nonetheless, that book made a bigger impression on me than anything else I read during my first year in college. I understood that, even if the book's examples were no longer timely, Huff's lessons were timeless. As the years went by, I found myself recalling How to Lie with Statistics when I encountered dubious numbers in my reading. I discovered that people continued to get confused by statistics in the same ways Huff had identified.

Updating Stat-Spotting has forced me to think about what's timely and what's timeless. This book offers a catalog of common problems that can be found in statistics, particularly the sorts of numbers that pop up when people are debating social issues. These problems - the errors in reasoning people make when they 
present statistics — don't go away; people keep making the same mistakes, sometimes because they don't know any better, sometimes because they're hoping to mislead an audience that isn't able to spot what's wrong with the numbers. These timeless lessonsknowing how to recognize some common ways statistics can be flawed-are what I hope you will take from this book.

The book also contains examples meant to illustrate each of the errors I describe. I chose most of these examples because they seemed timely (and, I hoped, engaging). Inevitably, as the years pass, these examples age; they no longer seem to have been "ripped from the headlines." So, given the opportunity to update Stat-Spotting, I had to decide what needed to be replaced.

I chose to make some selective changes: (I) I revised all the benchmark statistics because a benchmark that isn't up-to-date isn't that useful; (2) I updated the list of resources at the end of the book to give readers a better sense of where they could find current information; (3) I added an entire section on the rhetorical uses of statistics, complete with new problems to be spotted and new examples illustrating those problems; and (4) in a few cases where I knew the debate had evolved in important ways, I revised the discussion of an example or added newer, better references. However, most of the examples remain unchanged. They don't strike me as antiquated, and I continue to feel that a lot of them are pretty interesting. More importantly, I hope that the people who read this book will focus more on the timeless lessons and less on the timeliness of the examples. 